Relato de Caso

Case Report

André Vinicius Marcondes Natel Sales ${ }^{1}$

Célia Maria Giacheti ${ }^{1}$

Paula Cristina Cola $a^{1,2}$

Roberta Gonçalves da Silva ${ }^{1}$

Descritores

Deglutição

Transtorno de Deglutição

Síndrome de Down

Fluoroscopia

Análise Quantitativa

Software

Keywords

Swallowing

Deglutition Disorders

Down syndrome

Fluoroscopy

Quantitative Analysis

Software

Endereço para correspondência:

Roberta Gonçalves da Silva

Departamento de Fonoaudiologia,

Faculdade de Filosofia e Ciências,

Universidade Estadual Paulista -

UNESP

Avenida Higyno Muzzi Filho, 737,

Marília (SP), Brasil, CEP: 17525-900.

E-mail: rgsilva@marilia.unesp.br

Recebido em: Janeiro 26, 2017

Aceito em: Maio 23, 2017

\section{Análise qualitativa e quantitativa da deglutição orofaríngea na Síndrome de Down}

\author{
Qualitative and quantitative analysis of \\ oropharyngeal swallowing in Down syndrome
}

\section{RESUMO}

Objetivo: Este estudo tem por objetivo descrever a análise qualitativa e quantitativa temporal da deglutição orofaríngea em crianças com diagnóstico de Síndrome de Down (SD). Método: Estudo de série de seis casos, com idade variando de quatro a 17 meses (Média de 11,16 meses e mediana de 12 meses). Realizada análise qualitativa e quantitativa temporal da deglutição orofaríngea por meio de videofluoroscopia de deglutição e software específico. Foram analisados os parâmetros qualitativos de coordenação oral, resíduos orais, escape oral posterior, penetração, aspiração laringotraqueal e realizada análise do tempo de trânsito oral total (TTOT) e faríngeo. Resultados: Verificou-se alteração em pelo menos quatro dos parâmetros qualitativos investigados. Somente um dos indivíduos apresentou diferença no TTOT quando comparado com os demais e não houve diferença no tempo de trânsito faríngeo entre os casos. Conclusão: Houve alterações qualitativas na deglutição em crianças com SD e diferença no TTOT somente no caso de menor faixa etária.

Trabalho realizado no Laboratório de Disfagia, Programa de Pós-graduação em Fonoaudiologia, Universidade Estadual Paulista - UNESP - Marília (SP), Brasil.

${ }^{1}$ Universidade Estadual Paulista - UNESP - Marília (SP), Brasil.

${ }^{2}$ Universidade de Marília - UNIMAR - Marilia (SP), Brasil.

Fonte de financiamento: Fundação de Amparo ao Desenvolvimento da Pesquisa do Estado de São PauloFAPESP. Processo 2009/13779-0.

Conflito de interesses: nada a declarar. 


\section{INTRODUÇÃO}

A Síndrome de Down (SD) é uma doença genética causada pela trissomia do cromossomo $21 \mathrm{e}$ uma das principais causas de deficiência intelectual, com um a cada 600 a 800 nascimentos ${ }^{(1)}$. A síndrome possui seu fenótipo bem definido, conhecido e descrito pela literatura, com características físicas de fácil reconhecimento, identificação clínica e confirmação do diagnóstico genético.

No desempenho motor oral, encontram-se: presença de cavidade oral reduzida, hipotonia dos músculos orais e faciais, má oclusão dentária e macroglossia ${ }^{(1,2)}$. Além disso, estas crianças podem apresentar alterações no desenvolvimento do sistema nervoso central e na motilidade esofágica, acarretando prejuízos nas funções estomatognáticas, dentre elas a deglutição orofaríngea ${ }^{(2)}$.

Para a avaliação da deglutição, o exame de videofluoroscopia de deglutição (VFD), inclusive em pediatria, é considerado o método Gold Standard, possibilitando visualização de todas as fases e permitindo análises qualitativas e quantitativas de distância e tempo. Embora na SD alguns estudos tenham utilizado a VFD para análise qualitativa da deglutição orofaríngea, investigaram variáveis distintas das propostas neste estudo. Esses estudos fragmentaram a deglutição, alguns com maior ênfase na fase oral ${ }^{(3)}$ e outros na fase faríngea, destacando a presença ou ausência de aspiração traqueal ${ }^{(2)}$.

A análise quantitativa temporal da deglutição orofaríngea tem sido empregada na disfagia em casos pediátricos com menor frequência que a utilizada na população adultat ${ }^{(4)}$, com poucos estudos que investigaram crianças saudáveis ${ }^{(5)} \mathrm{e}$ em quadros patológicos como na paralisia cerebral. $\mathrm{Na} \mathrm{SD}$, não foram encontrados trabalhos com análise temporal mensurada por software conforme o aqui proposto.

Portanto, este estudo teve por objetivo descrever a análise qualitativa e quantitativa temporal da deglutição orofaríngea em crianças com SD.

\section{APRESENTAÇÃO DOS CASOS CLÍNICOS}

Estudo aprovado pelo Comitê de Ética em Pesquisa da Universidade Estadual Paulista-UNESP/Campus de Marília sob parecer número 1755/2009. Os responsáveis legais assinaram o Termo de Consentimento Livre e Esclarecido, consentindo, desta forma, com a realização e a divulgação da pesquisa e de seus resultados.
Para este estudo, foram incluídos seis exames videofluoroscópicos de deglutição de crianças com diagnóstico de SD que realizaram o exame em serviço especializado no período de 2015 a 2016. Para caracterização e descrição dos casos de SD confirmados por meio de exame laboratorial (cariótipo), foram coletadas as informações da investigação clínica, com levantamento de dados sobre idade, gênero, presença de queixas de alimentação e/ou deglutição, terapia fonoaudiológica, histórico de complicação pulmonar, presença de refluxo gastroesofágico (RGE), histórico de alteração cardíaca e necessidade de intervenção cardiocirúrgica descritos no Quadro 1.

Todos os indivíduos realizaram exame de videofluoroscopia de deglutição com a consistência líquida ofertada em mamadeira usual e em fluxo contínuo habitual de cada criança (o que não permite controle de volume), com visão lateral em posição sentada, com a cabeça disposta na direção do eixo corporal, sem flexão ou rotação, adaptando-se à sua postura, quando necessário.

Os exames foram analisados de forma qualitativa e quantitativa por dois juízes fonoaudiólogos com experiência na área. Depois da mensuração dos tempos de deglutição por cada julgador, foi realizada análise estatística para verificar a concordância entre eles com aplicação do teste $t$, considerando $\alpha<0,05$. Não houve diferença estatística significante entre os valores encontrados pelos julgadores $(\mathrm{p}=0,009)$. Foi realizada a média entre os julgadores para o tempo de trânsito oral e faríngeo. Para análise videofluoroscópica qualitativa, foram considerados: presença ou ausência de alteração na propulsão oral, alteração no vedamento do esfíncter labial, resíduos orais, escape oral posterior, penetração e aspiração laringotraqueal ${ }^{(6)}$. A análise quantitativa temporal da deglutição foi realizada por meio de software específico ${ }^{(7)}$ para mensurar, em milissegundos, o tempo de trânsito oral total (TTOT) e o tempo de trânsito faríngeo (TTF). Para os parâmetros qualitativos e quantitativos temporais estudados, foram considerados os critérios conforme descritos no Quadro 2.

Conforme apresentado na Tabela 1, observou-se que todos os casos apresentaram uma ou mais alterações qualitativas na fase oral da deglutição e que os casos 2 e 4 apresentaram alterações apenas na fase oral da deglutição. Os demais casos apresentaram comprometimento em ambas as fases, contudo a aspiração laringotraqueal só foi detectada no caso 6 .

$\mathrm{Na}$ análise do TTOT, somente o caso 6 apresentou valor discrepante quando comparado com os demais casos. Quanto ao TTF, todos os casos apresentaram valores dentro dos padrões de normalidade para a população infantil e conforme apresentado na Tabela 2.

Quadro 1. Descrição clínica dos casos

\begin{tabular}{|c|c|c|c|c|c|c|c|c|}
\hline \multirow[b]{2}{*}{ Caso } & \multirow[b]{2}{*}{ Idade } & \multirow[b]{2}{*}{ Gênero } & \multicolumn{6}{|c|}{ Histórico clínico } \\
\hline & & & $\begin{array}{l}\text { Queixas de } \\
\text { deglutição }\end{array}$ & $\begin{array}{l}\text { Complicação } \\
\text { pulmonar }\end{array}$ & $\begin{array}{c}\text { Refluxo } \\
\text { gastroesofágico }\end{array}$ & $\begin{array}{l}\text { Alteração } \\
\text { cardíaca }\end{array}$ & $\begin{array}{c}\text { Intervenção } \\
\text { Cardiocirúrgica }\end{array}$ & $\begin{array}{c}\text { Terapia } \\
\text { fonoaudiológica } \\
\text { prévia }\end{array}$ \\
\hline 1 & 1 a $5 \mathrm{~m}$ & Masculino & $\checkmark$ & & & $\checkmark$ & & $\checkmark$ \\
\hline 2 & $1 \mathrm{a} 2 \mathrm{~m}$ & Feminino & & $\checkmark$ & $\checkmark$ & $\checkmark$ & & $\checkmark$ \\
\hline 3 & $7 \mathrm{~m}$ & Masculino & $\checkmark$ & $\checkmark$ & $\checkmark$ & & & $\checkmark$ \\
\hline 4 & 1 a $3 \mathrm{~m}$ & Feminino & & $\checkmark$ & $\checkmark$ & $\checkmark$ & & $\checkmark$ \\
\hline 5 & $10 \mathrm{~m}$ & Feminino & $\checkmark$ & $\checkmark$ & $\checkmark$ & $\checkmark$ & $\checkmark$ & $\checkmark$ \\
\hline 6 & $4 \mathrm{~m}$ & Masculino & $\checkmark$ & $\checkmark$ & $\checkmark$ & $\checkmark$ & & $\checkmark$ \\
\hline
\end{tabular}

Legenda: $\checkmark=$ presente 
Quadro 2. Parâmetros qualitativos e quantitativo temporal da deglutição orofaríngea analisados na videofluoroscopia de deglutição

\begin{tabular}{|c|c|}
\hline Parâmetros & Descrição \\
\hline $\begin{array}{l}\text { Alteração no vedamento do } \\
\text { esfíncter labial }\end{array}$ & Incompleta oclusão entre o lábio e o bico da mamadeira com ou sem presença de escape oral anterior. \\
\hline Alteração na propulsão oral & Incoordenação das estruturas orais no transporte anteroposterior do bolo alimentar. \\
\hline Resíduo oral & Permanência de material contrastado na cavidade oral após a deglutição. \\
\hline Escape oral posterior & $\begin{array}{l}\text { Presença de escape oral prematuro do alimento para hipofaringe, considerando a posição do bolo alimentar onde se inicia } \\
\text { a resposta faríngea. }\end{array}$ \\
\hline Penetração & Presença de material contrastado dentro das estruturas laríngeas até acima da prega vocal ${ }^{(6)}$. \\
\hline Aspiração laringotraqueal & Presença de material contrastado abaixo das pregas vocais ${ }^{(6)}$. \\
\hline $\begin{array}{l}\text { Tempo de trânsito oral total } \\
\text { (TTOT) }\end{array}$ & $\begin{array}{l}\text { A definição de tempo de trânsito oral total (TTOT) foi descrita por Gatto et al. }{ }^{\left({ }^{(8)}\right.} \text { baseada no TTO proposto por Logemann et al. }{ }^{(9)} \text {. } \\
\text { O TTOT foi definido em milissegundos com início no primeiro frame, mostrando o alimento dentro da cavidade oral, e o } \\
\text { término no primeiro frame, mostrando a parte proximal do bolo alimentar na região final do palato duro e início do palato } \\
\text { mole (espinha nasal) até a margem inferior do ramo da mandíbula. }\end{array}$ \\
\hline Tempo de trânsito faríngeo & $\begin{array}{l}\text { A definição de tempo de trânsito faríngeo (TTF) foi baseada em Kendall et al. }{ }^{(10)} \text {. O TTF foi definido como início no primeiro } \\
\text { frame, mostrando a parte proximal do bolo alimentar na região final do palato duro e início do palato mole (espinha nasal) } \\
\text { até o ramo da mandíbula, e foi considerado término o momento em que o bolo alimentar passava pelo esfíncter esofágico } \\
\text { superior. Considerado normal o tempo de até } 1150 \mathrm{~ms}^{(5)} \text {. }\end{array}$ \\
\hline
\end{tabular}

Tabela 1. Frequência das alterações nos parâmetros qualitativos da deglutição

\begin{tabular}{|c|c|c|c|c|c|c|c|c|}
\hline \multirow[b]{2}{*}{ Caso } & \multicolumn{4}{|c|}{ Fase Oral } & \multicolumn{4}{|c|}{ Fase Faríngea } \\
\hline & $\begin{array}{l}\text { Alteração na } \\
\text { propulsão oral }\end{array}$ & $\begin{array}{l}\text { Alteração no } \\
\text { esfíncter labial }\end{array}$ & $\begin{array}{l}\text { Presença de } \\
\text { resíduos orais }\end{array}$ & Freq & $\begin{array}{l}\text { Escape } \\
\text { Oral } \\
\text { Posterior }\end{array}$ & Penetração & Aspiração & Freq \\
\hline 1 & + & - & + & $66,66 \%$ & + & + & - & $66,66 \%$ \\
\hline 2 & + & + & + & $100 \%$ & - & - & - & 0 \\
\hline 3 & + & + & + & $100 \%$ & + & - & - & $33,33 \%$ \\
\hline 4 & + & + & + & $100 \%$ & - & - & - & 0 \\
\hline 5 & + & + & + & $100 \%$ & + & + & - & $66,66 \%$ \\
\hline 6 & + & + & + & $100 \%$ & + & + & + & $100 \%$ \\
\hline Freq & $100 \%$ & $83,33 \%$ & $100 \%$ & & $66,66 \%$ & $50 \%$ & $16,66 \%$ & \\
\hline
\end{tabular}

Legenda: + = presente; - = ausente; Freq = frequência

Tabela 2. Frequência das alterações nos parâmetros qualitativos e na análise temporal de cada fase da deglutição

\begin{tabular}{ccccc}
\hline Caso & $\begin{array}{c}\text { Frequência de alterações } \\
\text { parâmetros qualitativos - fase } \\
\text { oral }\end{array}$ & $\begin{array}{c}\text { Frequência de alterações } \\
\text { parâmetros - fase faríngea }\end{array}$ & $\begin{array}{c}\text { Tempo de trânsito oral total } \\
\text { em milessegundos }\end{array}$ & $\begin{array}{c}\text { Tempo de trânsito faríngeo em } \\
\text { milessegundos }\end{array}$ \\
\hline 1 & ++ & ++ & 680 & 600 \\
2 & +++ & & 800 & 920 \\
3 & +++ & + & 550 & 590 \\
4 & +++ & & 890 & 720 \\
5 & +++ & ++ & 800 & 450 \\
6 & +++ & +++ & $2240^{*}$ & 440 \\
\hline
\end{tabular}

Legenda: Frequência de alterações qualitativas: Fase oral: $+++=100 \% ;++=66,66 \%$; Fase faríngea: $+++=75 \% ;++=50 \% ;+=25 \%$. ${ }^{*}$ TTOT com maior tempo da amostra

\section{DISCUSSÃO}

O fenótipo característico na facie, cavidade oral e cervical, associado à presença de hipotonia na SD, já foi apontado como um dos aspectos responsáveis pela presença de prejuízos para a deglutição orofaríngea nesta população ${ }^{(2)}$.

Neste estudo, constatou-se alteração na fase oral e faríngea da deglutição na maioria dos parâmetros qualitativos analisados. Esses resultados corroboram a literatura que já aponta que tanto as alterações anatômicas quanto o controle neuromotor da deglutição nesta população podem impactar o desempenho da deglutição conforme preconiza a literatura ${ }^{(3,11)}$.
Um aspecto relevante na discussão sobre as alterações qualitativas na fase oral da deglutição na SD deve-se à importância das áreas cerebrais na modulação da fase oral da deglutição. A literatura especializada preconiza que essa modulação ocorre com a ativação de diversas áreas corticais e subcorticais do sistema nervoso central. Desta forma, deve-se ressaltar que não somente a presença de alteração nos aspectos miofuncionais orofaciais da SD, mas também outras alterações em bases morfofisiológicas do sistema nervoso central poderiam contribuir para prejudicar a modulação da fase oral ${ }^{(12,13)}$.

A presença de penetração e aspiração laringotraqueal ocorreu somente em um dos casos e, embora seja um estudo com série 
de casos, observa-se que o relato de complicações pulmonares presentes na amostra foi muito maior que a frequência de aspiração. Neste estudo, a complicação pulmonar foi observada em cinco casos, conforme descrito no perfil da casuística, e os resultados apontaram presença de aspiração laringotraqueal em apenas um caso. Esse resultado pode sugerir que a complicação pulmonar presente nesta população não é resultado somente da presença de aspiração laringotraqueal e que questões como RGE e alterações cardíacas merecem ênfase na investigação da complicação pulmonar ${ }^{(13)}$. Na população pediátrica com diagnóstico de RGE, é comum a aspiração do conteúdo refluído ${ }^{(14)}$, gerando queixas referentes a engasgos durante a alimentação e que podem ser confundidas com os sintomas orofaríngeos. No entanto, considera-se relevante proceder à investigação objetiva da deglutição nesta população, uma vez que outros estudos com populações maiores já demonstraram maior frequência de aspiração ${ }^{(2,3)}$.

Quanto à análise quantitativa temporal da deglutição, verificou-se que somente o indivíduo mais jovem apresentou TTOT diferente dos demais e não foram encontradas alterações no TTF. Considerando que somente no TTOT houve muita diferença entre o indivíduo mais jovem da amostra e os demais, será necessário investigar o desempenho da deglutição quanto à maturação do SNC nesta população, uma vez que a diferença entre essa faixa etária e as demais é acentuada. Portanto, sugerimos considerar em estudos futuros sobre deglutição na SD a influência da idade sobre a biomecânica da deglutição, pois sabemos que o processo de maturação cerebral e o desenvolvimento orofacial ocasionam mudanças tanto nas estruturas quanto na coordenação dos mecanismos da deglutição, bem como na modulação oral, sendo que estas podem impactar diretamente esta função na população em geral, incluindo a da $\mathrm{SD}^{(14)}$.

Outro aspecto que deve ser considerado na mensuração do tempo das fases da deglutição, principalmente em Pediatria, é o utensílio utilizado para a oferta alimentar. Diferentes utensílios, tais como copo, mamadeira e colher, atuam de diferentes formas na modulação da fase oral da deglutição, podendo interferir no desempenho eficaz nas diferentes variáveis desta função, dentre eles nos tempos de trânsito ${ }^{(5,15)}$. No presente estudo, as mensurações foram realizadas mediante o uso da mamadeira e mostram que a presença do TTOT diferenciado, e aumentando até em relação ao padrão de normalidade de TTO citado na literatura para crianças ${ }^{(5)}$, não está relacionado com o utensílio, mas, provavelmente, com a faixa etária, por ter ocorrido somente no indivíduo mais jovem da amostra.

\section{COMENTÁRIOS FINAIS}

Entendemos que o tamanho amostral confere limitações a este estudo, porém as pesquisas com esta população e a deglutição ainda são de difícil realização, considerando que não é frequente o rastreio dos transtornos de alimentação e/ou deglutição ao nascimento e na infância nesta população. Além disso, muitos familiares não possuem queixas de engasgos e, assim, não são encaminhados para investigação precocemente. Quando as queixas referentes à mastigação aparecem, com o avanço da idade, as alterações na deglutição podem já ter sido compensadas e estão concentradas na fase oral da deglutição e na fase preparatória oral propriamente dita. Diante do exposto, sugerimos que a investigação das habilidades de deglutição seja realizada precocemente nesta população e possam contribuir para identificar os casos de maior risco. São necessários estudos futuros com maior número de criança com $\mathrm{SD}$ e distintas faixas etárias para facilitar a compreensão dos achados nesta importante doença genética.

\section{REFERÊNCIAS}

1. OMIM: Online Mendelian Inheritance in Man [Internet]. 2017 [citado em 2017 Jan 26]. Available from: http://omim.org/entry/190685.

2. O'Neill AC, Richter GT. Pharyngeal dysphagia in children with Down syndrome. Otolaryngol Head Neck Surg. 2013;149(1):146-50. PMid:23525851. http://dx.doi.org/10.1177/0194599813483445.

3. Hashimoto M, Igari K, Hanawa S, Ito A, Takahashi A, Ishida N, et al. Tongue pressure during swallowing in adults with down syndrome and its relationship with palatal morphology. Dysphagia. 2014;29(4):509-18. PMid:24844770. http://dx.doi.org/10.1007/s00455-014-9538-5.

4. Henderson M, Miles A, Holgate V, Peryman S, Allen J. Application and verification of quantitative objective videofluoroscopic swallowing measures in a pediatric population with Dysphagia. J Pediatr. 2016;178:200-5. PMid:27568657. http://dx.doi.org/10.1016/j.jpeds.2016.07.050.

5. Weckmueller J, Easterling C, Averdson J. Preliminary temporal measurement analysis of normal oropharyngeal swallowing in infants and young children. Dysphagia. 2011;26(2):135-43. PMid:20532920. http://dx.doi.org/10.1007/ s00455-010-9283-3.

6. Martin-Harris B, Brodsky MB, Michel Y, Castell DO, Schleicher M, Sandidge J, et al. MBS measurement tool for swallow impairment- MBSImp: establishing a standard. Dysphagia. 2008;23(4):392-405. PMid:18855050. http://dx.doi.org/10.1007/s00455-008-9185-9.

7. Spadotto AA, Gatto AR, Cola PC, Montagnoli NA, Schelp AO, Silva $\mathrm{RG}$, et al. Software para análise quantitativa da deglutição. Radiol Bras. 2008;41(1):25-8. http://dx.doi.org/10.1590/S0100-39842008000100008.

8. Gatto AR, Cola PC, Silva RG, Spadotto AA, Ribeiro PW, Schelp AO, et al. Sour taste and cold temperature in the oral phase of swallowing in patients after stroke. CoDAS. 2013;25(2):163-7. PMid:24408246. http://dx.doi. org/10.1590/S2317-17822013000200012.

9. Logemann JA, Pauloski BR, Coangelo L, Lazarus CL, Fujiu M, Kahrilas PJ. Effects of sour bolus on oropharyngeal swallowing measures in patients with neurogenic dysphagia. J Speech Hear Res. 1995;38(3):556-63. PMid:7674647. http://dx.doi.org/10.1044/jshr.3803.556.

10. Kendall KA, Leonard RJ, Mckenzie SW. Accomodation of changes in bolus viscosity in normal deglutition: a videofluoroscopic study. Ann Otol Rhinol Laryngol. 2001;110(11):1059-65. PMid:11713919. http://dx.doi.or $\mathrm{g} / 10.1177 / 000348940111001113$.

11. Durvasula VS, O'Neill AC, Richter GT. Oropharyngeal Dysphagia in children: mechanism, source, and management. Otolaryngol Clin North Am. 2014;47(5):691-720. PMid:25213278. http://dx.doi.org/10.1016/j. otc.2014.06.004.

12. Uppal H, Chandran S, Potluri R. Risk factors for mortality in Down Syndrome. J Intellect Disabil Res. 2015;59(9):873-81. PMid:25851193. http://dx.doi.org/10.1111/jir.12196.

13. Ling KH, Hewitt CA, Tan KL, Cheah PS, Vidyadaran S, Lai MI, et al. Functional transcriptome analysis of the postnatal brain of the Ts $1 \mathrm{Cje}$ mouse model for Down syndrome reveals global disruption of interferon-related molecular networks. BMC Genomics. 2014;22(15):624. PMid:25052193. http://dx.doi.org/10.1186/1471-2164-15-624. 
14. Breia P, Mendes R, Silvestre A, Gonçalves MJ, Figueira MJ, Bispo R. Adults with Down syndrome: characterization of a Portuguese sample. Acta Med Port. 2014;24(3):357-63. PMid:25017348. http://dx.doi.org/10.20344/amp.4898.

15. López CP, Chiari BM, Goulart AL, Furkim AM, Guedes ZC. Avaliação da deglutição em prematuros com mamadeira e copo. CoDAS. 2014;26(1):816. PMid:24714863.

\section{Contribuição dos autores}

AVMNS pesquisador principal, elaboração do estudo e cronograma, levantamento da literatura, coleta e análise dos dados, redação do artigo, submissão e trâmites do artigo; PCC, CMG e RGS orientadoras, elaboração da pesquisa, participaram da redação e correção do artigo bem como da aprovação da versão final. 